Acta Crystallographica Section E

Structure Reports

Online

ISSN 1600-5368

Peter Kirsop, John M. D. Storey and William T. A. Harrison*

Department of Chemistry, University of Aberdeen, Meston Walk, Aberdeen AB24 3UE, Scotland

Correspondence e-mail:

w.harrison@abdn.ac.uk

\section{Key indicators}

Single-crystal X-ray study

$T=120 \mathrm{~K}$

Mean $\sigma(\mathrm{C}-\mathrm{C})=0.006 \AA$

$R$ factor $=0.049$

$w R$ factor $=0.076$

Data-to-parameter ratio $=14.3$

For details of how these key indicators were automatically derived from the article, see http://journals.iucr.org/e.

\title{
4-Bromo-3,5-dihydroxybenzoic acid monohydrate
}

The crystal packing of the title compound, $\mathrm{C}_{7} \mathrm{H}_{5} \mathrm{BrO}_{4} \cdot \mathrm{H}_{2} \mathrm{O}$, is influenced by $\mathrm{O}-\mathrm{H} \cdots \mathrm{O}$ hydrogen bonds.

\section{Comment}

The title compound, (I) (Fig. 1), was prepared as part of our studies radical-mediated competitive cyclization reactions (Kirsop et al., 2007). Some crystal structures containing 4bromo-3,5-dihydroxybenzoic acid and its deprotonated anion in combination with 4,4-bipyridine derivatives have been described recently by Varughese \& Pedireddi (2006).<smiles>O=C(O)c1cc(O)c(Br)c(O)c1</smiles>

(I)

Compound (I) possesses normal geometric parameters (Allen et al., 1987). The dihedral angle between the mean plane of the $\mathrm{C} 1-\mathrm{C} 6$ benzne ring and the plane of the $\mathrm{C} 7 / \mathrm{O} 1 /$ $\mathrm{O} 2$ grouping is $4.5(5)^{\circ}$.

The crystal packing of (I) is influenced by $\mathrm{O}-\mathrm{H} \cdots \mathrm{O}$ hydrogen bonds (Table 1). First, classical inversion dimers involving $\mathrm{O} 4-\mathrm{H} 4 \cdots \mathrm{O} 3{ }^{\mathrm{iii}}$ and $\mathrm{O} 4{ }^{\mathrm{iii}}-\mathrm{H}^{\mathrm{iii}} \cdots \mathrm{O} 3$ bonds of

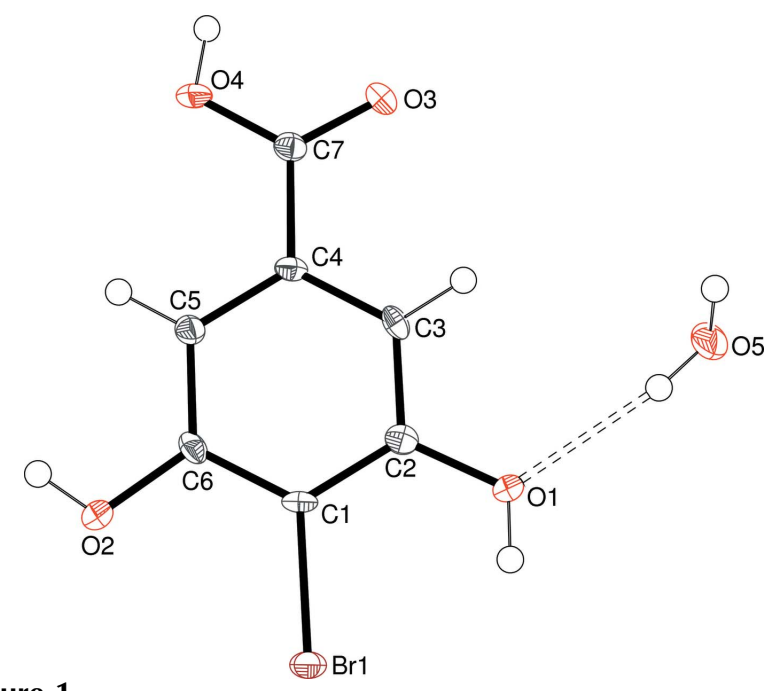

Figure 1

A view of the molecular structure of (I), showing $50 \%$ probability displacement ellipsoids for the non-hydrogen atoms. The hydrogen bond is indicated by a double-dashed line. 


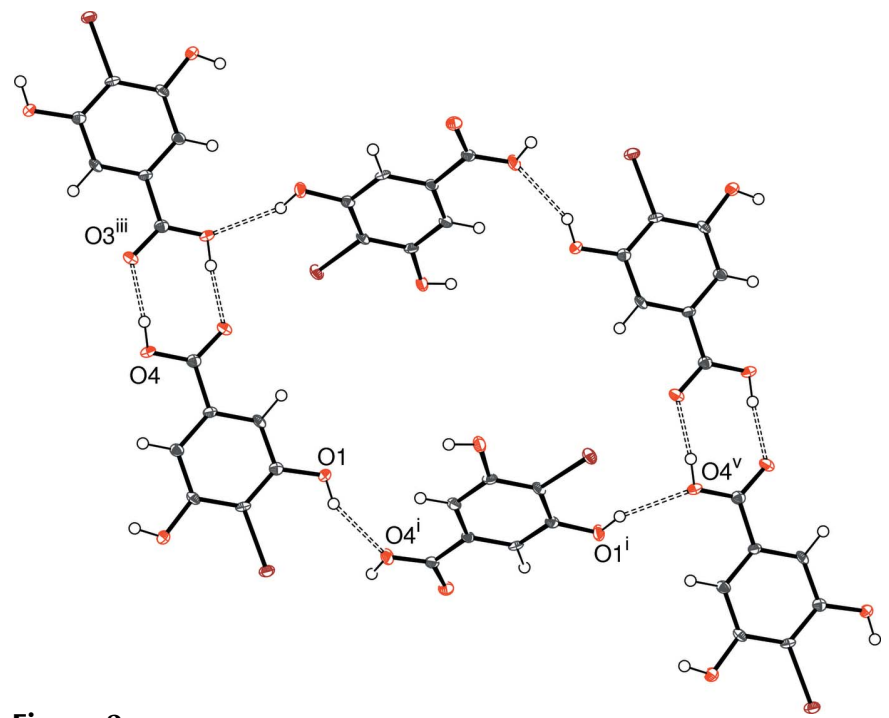

Figure 2

Part of the crystal structure of (I), showing an $R_{6}^{6}(32)$ supramolecular loop arising from the connectivity of six organic molecules. Dashed lines indicate hydrogen bonds. [Symmetry codes as in Table 1; additionally: (v) $x, y, z-1$.]

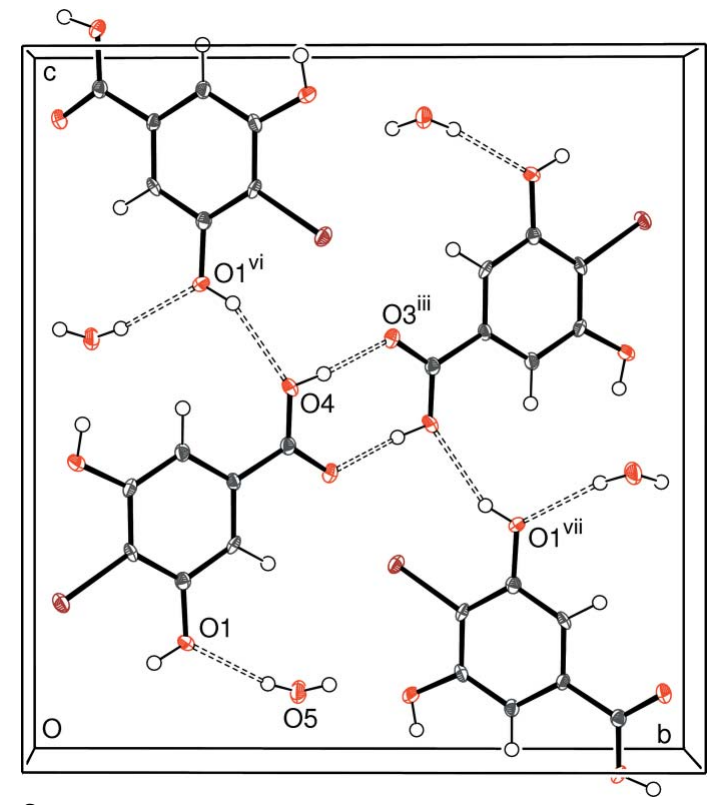

Figure 3

Unit-cell contents for (I), with $\mathrm{O}-\mathrm{H} \cdots \mathrm{O}$ hydrogen bonds indicated by double-dashed lines. [Symmetry codes as in Table 1; additionally: (vi) $x$, $\frac{1}{2}-y, \frac{1}{2}+z ;\left(\right.$ vii) $1-x, \frac{1}{2}+y, \frac{1}{2}-z$.]

adjacent head-to-head carboxylic acid groups are formed (Fig. 2) [symmetry code: (iii) $1-x, 1-y, 1-z$ ]. Atom H4 appeared to be well ordered in a difference map, but the C7O4 bond [1.288(5) $\AA$ ] is shorter and the $\mathrm{C} 7=\mathrm{O} 3$ bond [1.252 (5) $\AA$ ] is longer than expected for a well ordered carboxylic acid group: the mean $\mathrm{C}-\mathrm{O}$ and $\mathrm{C}=\mathrm{O}$ bond lengths in carboxylic acid groups bound to an aromatic ring are $1.226 \AA(\sigma=0.020 \AA)$ and $1.305 \AA(\sigma=0.020 \AA)$, respectively (Allen et al., 1987). This perhaps suggests partial disordering of $\mathrm{H} 4$ in (I), i.e. it is bound to both $\mathrm{O} 3$ and $\mathrm{O} 4$, but this was not visible in a difference map.
These dimeric pairs of 4-bromo-3,5-dihydroxybenzoic acid molecules are then linked into an infinite sheet by way of $\mathrm{O} 1-$ $\mathrm{H} 1 \cdots \mathrm{O} 4^{\mathrm{i}}$ bonds (Fig. 2). This results in distinctive $R_{6}^{6}(32)$ supramolecular loops (Bernstein et al., 1995). The O2-H2 hydroxyl group forms a hydrogen bond to a water molecule $\mathrm{O}$ atom. In turn, the water molecule acts as a donor for two more $\mathrm{O}-\mathrm{H} \cdots \mathrm{O}$ interactions, to result in a three-dimensional network (Fig. 3).

\section{Experimental}

A commercial sample of 4-bromo-3,5-dihydroxybenzoic acid was recrystallized from water, to result in slightly translucent needles of (I).

\section{Crystal data}

$\mathrm{C}_{7} \mathrm{H}_{5} \mathrm{BrO}_{4} \cdot \mathrm{H}_{2} \mathrm{O}$

$M_{r}=251.04$

Monoclinic, $P 2_{1} / c$

$a=3.7065$ (1) А

$b=14.4963$ (7)

$c=15.4548(8) \AA$

$\beta=94.209(3)^{\circ}$

$V=828.16(6) \AA^{3}$

\section{Data collection}

Nonius KappaCCD area-detector diffractometer

$\omega$ and $\varphi$ scans

Absorption correction: multi-scan (SADABS; Bruker, 2003)

$T_{\min }=0.588, T_{\max }=0.952$

Refinement

Refinement on $F^{2}$

$R\left[F^{2}>2 \sigma\left(F^{2}\right)\right]=0.049$

$w R\left(F^{2}\right)=0.076$

$S=1.08$

1900 reflections

133 parameters
$Z=4$

$D_{x}=2.013 \mathrm{Mg} \mathrm{m}^{-3}$

Mo $K \alpha$ radiation

$\mu=4.95 \mathrm{~mm}^{-1}$

$T=120(2) \mathrm{K}$

Needle, colourless

$0.12 \times 0.02 \times 0.01 \mathrm{~mm}$

9660 measured reflections 1900 independent reflections 1466 reflections with $I>2 \sigma(I)$ $R_{\text {int }}=0.085$

$\theta_{\max }=27.5^{\circ}$
$\mathrm{H}$ atoms treated by a mixture of independent and constrained refinement

$w=1 /\left[\sigma^{2}\left(F_{\mathrm{o}}^{2}\right)+1.4241 P\right]$

where $P=\left(F_{\mathrm{o}}{ }^{2}+2 F_{\mathrm{c}}{ }^{2}\right) / 3$

$(\Delta / \sigma)_{\max }<0.001$

$\Delta \rho_{\max }=0.65{\mathrm{e} \AA^{-3}}^{-3}$

$\Delta \rho_{\min }=-0.59{\mathrm{e} \AA^{-3}}^{-3}$
Table 1

Hydrogen-bond geometry $\left(\AA{ }^{\circ}\right)$.

\begin{tabular}{lllll}
\hline$D-\mathrm{H} \cdots A$ & $D-\mathrm{H}$ & $\mathrm{H} \cdots A$ & $D \cdots A$ & $D-\mathrm{H} \cdots A$ \\
\hline $\mathrm{O} 1-\mathrm{H} 1 \cdots \mathrm{O} 4^{\mathrm{i}}$ & $0.824(19)$ & $2.19(3)$ & $2.937(4)$ & $151(4)$ \\
$\mathrm{O} 2-\mathrm{H} 2 \cdots$ O $^{\mathrm{ii}}$ & $0.835(19)$ & $1.82(2)$ & $2.641(4)$ & $169(4)$ \\
$\mathrm{O}^{\mathrm{ii}}-\mathrm{H} 4 \cdots 3^{\text {iii }}$ & $0.834(19)$ & $1.79(2)$ & $2.620(4)$ & $175(5)$ \\
$\mathrm{O} 5-\mathrm{H} 6 \cdots \mathrm{O} 2^{\text {iv }}$ & $0.828(19)$ & $2.18(3)$ & $2.918(4)$ & $149(5)$ \\
$\mathrm{O} 5-\mathrm{H} 7 \cdots \mathrm{O} 1$ & $0.840(19)$ & $2.11(2)$ & $2.919(4)$ & $163(4)$ \\
\hline Symmetry codes: & (i) $x,-y+\frac{1}{2}, z-\frac{1}{2}$; (ii) & $x+1,-y+\frac{1}{2}, z+\frac{1}{2} ; \quad$ (iii) \\
$-x+1,-y+1,-z+1 ;$ (iv) $-x+2, y+\frac{1}{2},-z+\frac{1}{2}$. & &
\end{tabular}

O-bound $\mathrm{H}$ atoms were located in a difference map and their positions were refined with the distance restraint $\mathrm{O}-\mathrm{H}=0.85$ (2) $\AA$. $U_{\text {iso }}(\mathrm{H})$ was set equal to $1.2 U_{\text {eq }}(\mathrm{O})$. C-bound $\mathrm{H}$ atoms were placed in idealized positions, with $\mathrm{C}-\mathrm{H}=0.95 \AA$, and refined as riding, with $U_{\text {iso }}(\mathrm{H})=1.2 U_{\text {eq }}(\mathrm{C})$.

Data collection: COLLECT (Nonius, 1998); cell refinement: SCALEPACK (Otwinowski \& Minor, 1997); data reduction: SCALEPACK, DENZO (Otwinowski \& Minor, 1997) and SORTAV (Blessing, 1995); program(s) used to solve structure: SHELXS97 (Sheldrick, 1997); program(s) used to refine structure: SHELXL97 


\section{organic papers}

(Sheldrick, 1997); molecular graphics: ORTEP-3 (Farrugia, 1997); software used to prepare material for publication: SHELXL97.

The authors thank the EPSRC's UK National Crystallography Service, University of Southampton, for the data collection.

\section{References}

Allen, F. H., Kennard, O., Watson, D. G., Brammer, L., Orpen, A. G. \& Taylor, R. (1987). J. Chem. Soc. Perkin Trans. 2, pp. S1-19.
Bernstein, J., Davis, R. E., Shimoni, L. \& Chang, N.-L. (1995). Angew. Chem. Int. Ed. Engl. 34, 1555-1573.

Blessing, R. H. (1995). Acta Cryst. A51, 33-38.

Bruker (2003). SADABS. Bruker AXS Inc., Madison, Wisconsin, USA.

Farrugia, L. J. (1997). J. Appl. Cryst. 30, 565.

Kirsop, P., Storey, J. M. D. \& Harrison, W. T. A. (2007). Acta Cryst. E63, o8330835 .

Nonius (1998). COLLECT. Nonius BV, Delft, The Netherlands.

Otwinowski, Z. \& Minor, W. (1997). Methods in Enzymology, Vol. 276, Macromolecular Crystallography, Part A, edited by C. W. Carter Jr \& R. M. Sweet, pp. 307-326. New York: Academic Press.

Sheldrick, G. M. (1997). SHELXS97 and SHELXL97. University of Göttingen, Germany.

Varughese, S. \& Pedireddi, V. R. (2006). Chem. Eur. J. 12, 1597-1609. 


\title{
supporting information
}

Acta Cryst. (2007). E63, o1441-o1443 [https://doi.org/10.1107/S1600536807003492]

\section{4-Bromo-3,5-dihydroxybenzoic acid monohydrate}

\author{
Peter Kirsop, John M. D. Storey and William T. A. Harrison
}

4-Bromo-3,5-dihydroxybenzoic acid monohydrate

Crystal data

$\mathrm{C}_{7} \mathrm{H}_{5} \mathrm{BrO}_{4} \cdot \mathrm{H}_{2} \mathrm{O}$

$M_{r}=251.04$

Monoclinic, $P 2_{1} / c$

Hall symbol: $-\mathrm{P} 2 \mathrm{ybc}$

$a=3.7065(1) \AA$

$b=14.4963(7) \AA$

$c=15.4548(8) \AA$

$\beta=94.209(3)^{\circ}$

$V=828.16(6) \AA^{3}$

$Z=4$

\section{Data collection}

Nonius KappaCCD area-detector diffractometer

Radiation source: fine-focus sealed tube Graphite monochromator

$\omega$ and $\varphi$ scans

Absorption correction: multi-scan

(SADABS; Bruker, 2003)

$T_{\min }=0.588, T_{\max }=0.952$

\section{Refinement}

Refinement on $F^{2}$

Least-squares matrix: full

$R\left[F^{2}>2 \sigma\left(F^{2}\right)\right]=0.049$

$w R\left(F^{2}\right)=0.076$

$S=1.08$

1900 reflections

133 parameters

6 restraints

Primary atom site location: structure-invariant direct methods
$F(000)=496$

$D_{\mathrm{x}}=2.013 \mathrm{Mg} \mathrm{m}^{-3}$

Mo $K \alpha$ radiation, $\lambda=0.71073 \AA$

Cell parameters from 1924 reflections

$\theta=2.9-27.5^{\circ}$

$\mu=4.95 \mathrm{~mm}^{-1}$

$T=120 \mathrm{~K}$

Needle, colourless

$0.12 \times 0.02 \times 0.01 \mathrm{~mm}$

9660 measured reflections

1900 independent reflections

1466 reflections with $I>2 \sigma(I)$

$R_{\text {int }}=0.085$

$\theta_{\max }=27.5^{\circ}, \theta_{\min }=3.0^{\circ}$

$h=-4 \rightarrow 4$

$k=-18 \rightarrow 18$

$l=-20 \rightarrow 19$

Secondary atom site location: difference Fourier map

Hydrogen site location: difmap and geom

$\mathrm{H}$ atoms treated by a mixture of independent and constrained refinement

$w=1 /\left[\sigma^{2}\left(F_{\mathrm{o}}^{2}\right)+1.4241 P\right]$

where $P=\left(F_{\mathrm{o}}{ }^{2}+2 F_{\mathrm{c}}{ }^{2}\right) / 3$

$(\Delta / \sigma)_{\max }<0.001$

$\Delta \rho_{\max }=0.65$ e $\AA^{-3}$

$\Delta \rho_{\min }=-0.59$ e $\AA^{-3}$

Special details

Geometry. All e.s.d.'s (except the e.s.d. in the dihedral angle between two 1.s. planes) are estimated using the full covariance matrix. The cell e.s.d.'s are taken into account individually in the estimation of e.s.d.'s in distances, angles and torsion angles; correlations between e.s.d.'s in cell parameters are only used when they are defined by crystal symmetry. An approximate (isotropic) treatment of cell e.s.d.'s is used for estimating e.s.d.'s involving 1.s. planes. 
Refinement. Refinement of $F^{2}$ against ALL reflections. The weighted $R$-factor $w R$ and goodness of fit $S$ are based on $F^{2}$, conventional $R$-factors $R$ are based on $F$, with $F$ set to zero for negative $F^{2}$. The threshold expression of $F^{2}>\sigma\left(F^{2}\right)$ is used only for calculating $R$-factors(gt) etc. and is not relevant to the choice of reflections for refinement. $R$-factors based on $F^{2}$ are statistically about twice as large as those based on $F$, and $R$ - factors based on ALL data will be even larger.

Fractional atomic coordinates and isotropic or equivalent isotropic displacement parameters $\left(\hat{A}^{2}\right)$

\begin{tabular}{lllll}
\hline & $x$ & $y$ & $z$ & $U_{\text {iso }} * / U_{\text {eq }}$ \\
\hline C1 & $0.9836(10)$ & $0.1596(3)$ & $0.3025(3)$ & $0.0126(9)$ \\
C2 & $0.8179(9)$ & $0.2346(3)$ & $0.2596(3)$ & $0.0130(9)$ \\
C3 & $0.7138(10)$ & $0.3092(3)$ & $0.3078(3)$ & $0.0130(9)$ \\
H3 & 0.5971 & 0.3604 & 0.2795 & $0.016^{*}$ \\
C4 & $0.7790(9)$ & $0.3094(3)$ & $0.3969(3)$ & $0.0120(9)$ \\
C5 & $0.9462(10)$ & $0.2347(3)$ & $0.4396(3)$ & $0.0138(9)$ \\
H5 & 0.9906 & 0.2355 & 0.5010 & $0.017^{*}$ \\
C6 & $1.0481(10)$ & $0.1587(3)$ & $0.3922(3)$ & $0.0136(9)$ \\
C7 & $0.6600(10)$ & $0.3905(3)$ & $0.4454(3)$ & $0.0138(9)$ \\
O1 & $0.7523(8)$ & $0.23889(19)$ & $0.1721(2)$ & $0.0193(7)$ \\
H1 & $0.798(11)$ & $0.192(2)$ & $0.145(3)$ & $0.023^{*}$ \\
O2 & $1.2073(7)$ & $0.08285(19)$ & $0.42874(19)$ & $0.0185(7)$ \\
H2 & $1.261(11)$ & $0.092(3)$ & $0.4814(14)$ & $0.022^{*}$ \\
O3 & $0.4904(7)$ & $0.45364(18)$ & $0.40498(19)$ & $0.0182(7)$ \\
O4 & $0.7399(8)$ & $0.39319(19)$ & $0.5279(2)$ & $0.0208(7)$ \\
H4 & $0.668(11)$ & $0.4435(19)$ & $0.546(3)$ & $0.025^{*}$ \\
Br1 & $1.13095(10)$ & $0.05915(3)$ & $0.23622(3)$ & $0.01626(13)$ \\
O5 & $0.4339(8)$ & $0.4063(2)$ & $0.0945(2)$ & $0.0259(8)$ \\
H6 & $0.559(10)$ & $0.4521(19)$ & $0.108(3)$ & $0.031^{*}$ \\
H7 & $0.561(10)$ & $0.360(2)$ & $0.110(3)$ & $0.031^{*}$ \\
& & & &
\end{tabular}

Atomic displacement parameters $\left(\AA^{2}\right)$

\begin{tabular}{lllllll}
\hline & $U^{11}$ & $U^{22}$ & $U^{33}$ & $U^{12}$ & $U^{13}$ & $U^{23}$ \\
\hline $\mathrm{C} 1$ & $0.0125(18)$ & $0.009(2)$ & $0.016(2)$ & $0.0006(15)$ & $0.0028(17)$ & $-0.0048(17)$ \\
$\mathrm{C} 2$ & $0.0119(19)$ & $0.0117(19)$ & $0.016(2)$ & $-0.0020(15)$ & $0.0017(17)$ & $-0.0004(19)$ \\
$\mathrm{C} 3$ & $0.0139(18)$ & $0.010(2)$ & $0.016(2)$ & $-0.0010(15)$ & $0.0024(17)$ & $0.0059(18)$ \\
$\mathrm{C} 4$ & $0.0131(18)$ & $0.009(2)$ & $0.014(2)$ & $-0.0002(15)$ & $0.0047(17)$ & $-0.0015(17)$ \\
$\mathrm{C} 5$ & $0.0149(19)$ & $0.011(2)$ & $0.015(3)$ & $-0.0008(16)$ & $-0.0021(17)$ & $-0.0003(18)$ \\
$\mathrm{C} 6$ & $0.0153(19)$ & $0.009(2)$ & $0.016(2)$ & $-0.0005(15)$ & $-0.0003(17)$ & $0.0036(18)$ \\
C7 & $0.0142(19)$ & $0.011(2)$ & $0.016(3)$ & $-0.0023(16)$ & $0.0020(17)$ & $-0.0001(18)$ \\
O1 & $0.0331(16)$ & $0.0136(16)$ & $0.0111(18)$ & $0.0046(13)$ & $0.0005(13)$ & $-0.0024(13)$ \\
O2 & $0.0272(15)$ & $0.0150(16)$ & $0.0127(17)$ & $0.0028(12)$ & $-0.0032(13)$ & $-0.0031(13)$ \\
O3 & $0.0242(14)$ & $0.0116(16)$ & $0.0184(18)$ & $0.0038(12)$ & $-0.0005(13)$ & $0.0024(13)$ \\
O4 & $0.0350(17)$ & $0.0121(16)$ & $0.0154(19)$ & $0.0052(13)$ & $0.0027(14)$ & $-0.0045(14)$ \\
Br1 & $0.0178(2)$ & $0.0136(2)$ & $0.0172(2)$ & $0.00327(18)$ & $-0.00002(15)$ & $-0.0047(2)$ \\
O5 & $0.0401(19)$ & $0.0128(16)$ & $0.023(2)$ & $-0.0043(13)$ & $-0.0081(16)$ & $0.0011(15)$ \\
& & & & & &
\end{tabular}


Geometric parameters $\left(\AA,{ }^{\circ}\right)$

\begin{tabular}{|c|c|c|c|}
\hline $\mathrm{C} 1-\mathrm{C} 6$ & $1.388(6)$ & $\mathrm{C} 5-\mathrm{H} 5$ & 0.9500 \\
\hline $\mathrm{C} 1-\mathrm{C} 2$ & $1.393(5)$ & $\mathrm{C} 6-\mathrm{O} 2$ & $1.352(5)$ \\
\hline $\mathrm{C} 1-\mathrm{Br} 1$ & $1.885(4)$ & $\mathrm{C} 7-\mathrm{O} 3$ & $1.252(5)$ \\
\hline $\mathrm{C} 2-\mathrm{O} 1$ & $1.356(5)$ & $\mathrm{C} 7-\mathrm{O} 4$ & $1.288(5)$ \\
\hline $\mathrm{C} 2-\mathrm{C} 3$ & $1.384(5)$ & $\mathrm{O} 1-\mathrm{H} 1$ & $0.824(19)$ \\
\hline $\mathrm{C} 3-\mathrm{C} 4$ & $1.381(6)$ & $\mathrm{O} 2-\mathrm{H} 2$ & $0.835(19)$ \\
\hline $\mathrm{C} 3-\mathrm{H} 3$ & 0.9500 & $\mathrm{O} 4-\mathrm{H} 4$ & $0.834(19)$ \\
\hline $\mathrm{C} 4-\mathrm{C} 5$ & $1.390(5)$ & $\mathrm{O} 5-\mathrm{H} 6$ & $0.828(19)$ \\
\hline $\mathrm{C} 4-\mathrm{C} 7$ & $1.479(5)$ & $\mathrm{O} 5-\mathrm{H} 7$ & $0.840(19)$ \\
\hline $\mathrm{C} 5-\mathrm{C} 6$ & $1.391(5)$ & & \\
\hline $\mathrm{C} 6-\mathrm{C} 1-\mathrm{C} 2$ & $121.4(4)$ & $\mathrm{C} 4-\mathrm{C} 5-\mathrm{H} 5$ & 120.2 \\
\hline $\mathrm{C} 6-\mathrm{C} 1-\mathrm{Br} 1$ & $120.0(3)$ & $\mathrm{C} 6-\mathrm{C} 5-\mathrm{H} 5$ & 120.2 \\
\hline $\mathrm{C} 2-\mathrm{C} 1-\mathrm{Br} 1$ & $118.6(3)$ & $\mathrm{O} 2-\mathrm{C} 6-\mathrm{C} 1$ & $117.6(4)$ \\
\hline $\mathrm{O} 1-\mathrm{C} 2-\mathrm{C} 3$ & $117.6(4)$ & $\mathrm{O} 2-\mathrm{C} 6-\mathrm{C} 5$ & $123.4(4)$ \\
\hline $\mathrm{O} 1-\mathrm{C} 2-\mathrm{C} 1$ & 123.5 & $\mathrm{C} 1-\mathrm{C} 6-\mathrm{C} 5$ & $119.0(4)$ \\
\hline $\mathrm{C} 3-\mathrm{C} 2-\mathrm{C} 1$ & $118.9(4)$ & $\mathrm{O} 3-\mathrm{C} 7-\mathrm{O} 4$ & $122.9(4)$ \\
\hline $\mathrm{C} 4-\mathrm{C} 3-\mathrm{C} 2$ & $120.2(4)$ & $\mathrm{O} 3-\mathrm{C} 7-\mathrm{C} 4$ & $119.1(4)$ \\
\hline $\mathrm{C} 4-\mathrm{C} 3-\mathrm{H} 3$ & 119.9 & $\mathrm{O} 4-\mathrm{C} 7-\mathrm{C} 4$ & $118.0(4)$ \\
\hline $\mathrm{C} 2-\mathrm{C} 3-\mathrm{H} 3$ & 119.9 & $\mathrm{C} 2-\mathrm{O} 1-\mathrm{H} 1$ & $116(3)$ \\
\hline $\mathrm{C} 3-\mathrm{C} 4-\mathrm{C} 5$ & $120.8(4)$ & $\mathrm{C} 6-\mathrm{O} 2-\mathrm{H} 2$ & $110(3)$ \\
\hline $\mathrm{C} 3-\mathrm{C} 4-\mathrm{C} 7$ & 118.0 & $\mathrm{C} 7-\mathrm{O} 4-\mathrm{H} 4$ & $108(3)$ \\
\hline $\mathrm{C} 5-\mathrm{C} 4-\mathrm{C} 7$ & $121.2(4)$ & $\mathrm{H} 6-\mathrm{O} 5-\mathrm{H} 7$ & $106(3)$ \\
\hline $\mathrm{C} 4-\mathrm{C} 5-\mathrm{C} 6$ & $119.7(4)$ & & \\
\hline $\mathrm{C} 6-\mathrm{C} 1-\mathrm{C} 2-\mathrm{O} 1$ & $179.4(3)$ & $\mathrm{C} 2-\mathrm{C} 1-\mathrm{C} 6-\mathrm{O} 2$ & $179.4(3)$ \\
\hline $\mathrm{Br} 1-\mathrm{C} 1-\mathrm{C} 2-\mathrm{O} 1$ & $0.8(5)$ & $\mathrm{Br} 1-\mathrm{C} 1-\mathrm{C} 6-\mathrm{O} 2$ & $-2.0(5)$ \\
\hline $\mathrm{C} 6-\mathrm{C} 1-\mathrm{C} 2-\mathrm{C} 3$ & $-0.5(5)$ & $\mathrm{C} 2-\mathrm{C} 1-\mathrm{C} 6-\mathrm{C} 5$ & $-0.3(6)$ \\
\hline $\mathrm{Br} 1-\mathrm{C} 1-\mathrm{C} 2-\mathrm{C} 3$ & $-179.1(3)$ & $\mathrm{Br} 1-\mathrm{C} 1-\mathrm{C} 6-\mathrm{C} 5$ & $178.2(3)$ \\
\hline $\mathrm{O} 1-\mathrm{C} 2-\mathrm{C} 3-\mathrm{C} 4$ & $-179.0(3)$ & $\mathrm{C} 4-\mathrm{C} 5-\mathrm{C} 6-\mathrm{O} 2$ & $-179.1(3)$ \\
\hline $\mathrm{C} 1-\mathrm{C} 2-\mathrm{C} 3-\mathrm{C} 4$ & $1.0(5)$ & $\mathrm{C} 4-\mathrm{C} 5-\mathrm{C} 6-\mathrm{C} 1$ & $0.7(5)$ \\
\hline $\mathrm{C} 2-\mathrm{C} 3-\mathrm{C} 4-\mathrm{C} 5$ & $-0.6(5)$ & $\mathrm{C} 3-\mathrm{C} 4-\mathrm{C} 7-\mathrm{O} 3$ & $3.4(5)$ \\
\hline $\mathrm{C} 2-\mathrm{C} 3-\mathrm{C} 4-\mathrm{C} 7$ & $-179.6(3)$ & $\mathrm{C} 5-\mathrm{C} 4-\mathrm{C} 7-\mathrm{O} 3$ & $-175.5(3)$ \\
\hline $\mathrm{C} 3-\mathrm{C} 4-\mathrm{C} 5-\mathrm{C} 6$ & $-0.2(5)$ & $\mathrm{C} 3-\mathrm{C} 4-\mathrm{C} 7-\mathrm{O} 4$ & $-175.9(3)$ \\
\hline $\mathrm{C} 7-\mathrm{C} 4-\mathrm{C} 5-\mathrm{C} 6$ & $178.7(3)$ & $\mathrm{C} 5-\mathrm{C} 4-\mathrm{C} 7-\mathrm{O} 4$ & $5.1(5)$ \\
\hline
\end{tabular}

Hydrogen-bond geometry $\left(\AA,{ }^{\circ}\right)$

\begin{tabular}{lllll}
\hline$D-\mathrm{H} \cdots A$ & $D-\mathrm{H}$ & $\mathrm{H} \cdots A$ & $D \cdots A$ & $D-\mathrm{H} \cdots A$ \\
\hline $\mathrm{O} 1-\mathrm{H} 1 \cdots \mathrm{O} 4^{\mathrm{i}}$ & $0.82(2)$ & $2.19(3)$ & $2.937(4)$ & $151(4)$ \\
$\mathrm{O} 2-\mathrm{H} 2 \cdots 5^{\mathrm{ii}}$ & $0.84(2)$ & $1.82(2)$ & $2.641(4)$ & $169(4)$ \\
$\mathrm{O} 4-\mathrm{H} 4 \cdots \mathrm{O} 3^{\mathrm{iii}}$ & $0.83(2)$ & $1.79(2)$ & $2.620(4)$ & $175(5)$ \\
$\mathrm{O} 5-\mathrm{H} 6 \cdots \mathrm{O} 2^{\mathrm{iv}}$ & $0.83(2)$ & $2.18(3)$ & $2.918(4)$ & $149(5)$ \\
$\mathrm{O} 5-\mathrm{H} 7 \cdots \mathrm{O} 1$ & $0.84(2)$ & $2.11(2)$ & $2.919(4)$ & $163(4)$
\end{tabular}

Symmetry codes: (i) $x,-y+1 / 2, z-1 / 2$; (ii) $x+1,-y+1 / 2, z+1 / 2$; (iii) $-x+1,-y+1,-z+1$; (iv) $-x+2, y+1 / 2,-z+1 / 2$. 